Internist 2007 • 48:489-496

DOI 10.1007/s00108-007-1814-0

Online publiziert: 1. März 2007

(c) Springer Medizin Verlag 2007

Schwerpunktherausgeber M. Battegay, Basel/Schweiz

B. Zabel, Borstel

G. Laifer ${ }^{1} \cdot$ S. Bassetti ${ }^{2}$

${ }^{1}$ Klinik für Infektiologie, Universitätsspital Basel

${ }^{2}$ Medizinische Klinik, Kantonsspital Olten

\title{
Diagnostik der pulmonalen Tuberkulose beim Erwachsenen
}

tierend und wird oft nicht diagnostiziert. In den folgenden 2 Jahren entwickeln etwa $5 \%$ der Infizierten eine aktive TB, weitere $5 \%$ im Laufe des Lebens. 90\% aller Immunkompetenten werden keine klinisch manifeste Erkrankung durchmachen [19]. Bei ihnen liegt eine latente Tuberkulose vor. Bei einer HIV-Koinfektion liegt diese Progressionsrate deutlich höher (ca. 8\%/Jahr).

Sonderformen sind die Miliartuberkulose und das Tuberkulom. Unter Miliartuberkulose werden alle progredienten hämatogen disseminierten Formen zusammengefasst, welche akut oder - v. a. bei älteren Menschen - verzögert verlaufen können. Ein Tuberkulom entsteht entweder im Rahmen der Primärinfektion oder als abgekapselte Reaktivierung. Es ist in der Regel asymptomatisch, kann sich jedoch radiologisch wie ein Karzinom oder Metastasen präsentieren. Im Folgenden beschränken wir uns auf die Diagnostik der latenten und der pulmonalen Tuberkulose beim Erwachsenen.

\section{Diagnostik der latenten Tuberkulose (Tuberkulintest und IGRAs)}

Der Tuberkulintest misst eine zelluläre Immunantwort vom Typ der verzögerten Hypersensitivität. Die Injektion (2 Tuberkulineinheiten gereinigtes Tuberkulin PPD-RT-23, welche 5 Einheiten des in den USA verwendeten Tuberkulin PPD entsprechen) erfolgt intrakutan an der Beugeseite des Unterarms (Mantoux-Test), tiefere Injektionen führen zu falsch-negativen Resultaten. Die Ablesung erfolgt nach 48 bis 72 Stunden (spätestens nach 7 Tagen) durch Ausmessen der Induration in der Querachse des Unterarms. Nur Personen mit einem hohen TB-Risiko, erhöhtem beruflichen Risiko (z. B. Angestellte im Gesundheitswesen) und Kontaktpertion über mehrere Stunden in schlecht gelüfteten Räumen zur Übertragung nötig ist, kurze Kontakte oder Kontakte im Freien sind meist nicht ausreichend. Überleben die Mykobakterien die initiale Abwehr, so replizieren sie sich langsam innerhalb der Makrophagen. Die zelluläre Immunität setzt erst nach 3 bis 9 Wochen ein und ist messbar mit dem Tuberkulintest. Vor dieser Zeit können Mykobakterien vom initialen Fokus lymphogen zu regionalen Lymphknoten und hämatogen in andere Gewebe und Organe gelangen und dort einen Fokus für eine spätere extrapulmonale Erkrankung bilden. Die primäre TB ist meist selbstlimi-

Abb. 1 Typisches Oberlappeninfiltrat bei pulmonaler Tuberkulose

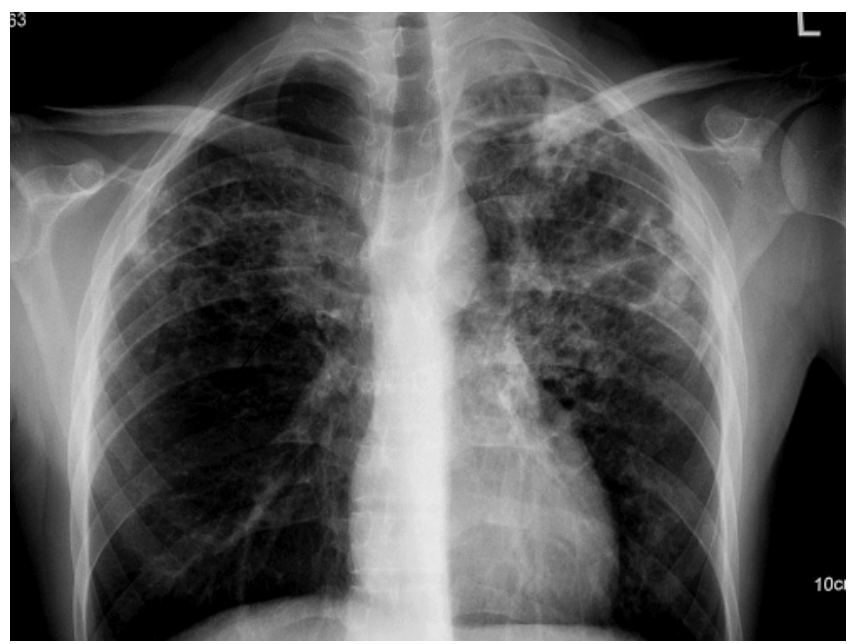




\begin{tabular}{|c|c|}
\hline Induration & Personengruppen, bei denen der Test als positiv interpretiert wird \\
\hline \multirow[t]{5}{*}{$\geq 5 \mathrm{~mm}$} & HIV-Positive \\
\hline & Naher Kontakt ( $\leq 2$ Jahre) zu Indexpatienten mit offener Lungentuberkulose \\
\hline & $\begin{array}{l}\text { Radiologische Residuen einer unbehandelten TB nach Ausschluss einer aktiven } \\
\text { Erkrankung }\end{array}$ \\
\hline & Immunsupprimierte: $\geq 15$ mg Prednison/Tag $\geq 1$ Monat; nach Organtransplantation \\
\hline & Personen unter (oder bei geplanten) anti-TNF-a-Antikörpern \\
\hline \multirow[t]{6}{*}{$\geq 10 \mathrm{~mm}$} & Immigranten $<5$ Jahre nach Einreise aus einem Hochendemiegebiet \\
\hline & $\begin{array}{l}\text { Personen mit erhöhtem TB-Risiko: Silikose, terminale Niereninsuffizienz, Malnutri- } \\
\text { tion, Diabetes mellitus, HNO- oder Lungenmalignome, Lymphom, Leukämie, Ge- } \\
\text { wichtsverlust } \geq 10 \% \text {, St. n. Gastrektomie oder ileojejunaler Bypasschirurgie, Immun- } \\
\text { suppression }<15 \text { mg Prednison/Tag }\end{array}$ \\
\hline & Intravenöse Drogenkonsumenten \\
\hline & Mitarbeiter in Gesundheitswesen, Mykobakterienlabor oder Pflegeheimen \\
\hline & Kinder < 4 Jahren \\
\hline & Dokumentierte Tuberkulinzunahme $\geq 10 \mathrm{~mm}$ innerhalb 2 Jahren \\
\hline$\geq 15 \mathrm{~mm}$ & Alle anderen (diese Personen sollten ohne Indikation nicht gescreent werden) \\
\hline
\end{tabular}

sonen im Rahmen einer Umgebungsuntersuchung sollten getestet werden [1] Praktisch alle Personen mit einer Induration $>15 \mathrm{~mm}$ und $90 \%$ mit einer Induration $\geq 10 \mathrm{~mm}$ sind infiziert mit $M$. tuberculosis. Ein Problem bleibt die Interpretation bei BCG-geimpften Personen in Niedrigprävalenzländern: Testresultate bis $18 \mathrm{~mm}$ bei <40-Jährigen sind eher Folge der Impfung und sollten nicht ohne weitere Argumente zu einer präventiven Therapie führen [21]. Interpretationsrichtlinien sind in - Tab. 1 aufgelistet. Die Wiederholung des Tuberkulintests eine bis wenige Wochen nach dem ersten Test kann vor allem bei Erwachsenen zu einer Vergrößerung der Reaktion auf Tuberkulin führen. Dabei handelt es sich um eine Reaktivierung einer früheren Immunität („Boostereffekt“', z. B. nach BCG-Impfung oder durchgemachter TB-Infektion). Bei Umgebungsuntersuchungen oder falls repetitive Tests erfolgen (z. B. expositionsgefährdete Berufe) wird deshalb empfohlen, Personen mit einem negativen Test nach einer Woche erneut zu testen. Das Resultat dieses zweiten Tests gilt als Ausgangswert für Verlaufstestungen. Diese Personen haben jedoch ein niedriges Risiko und werden behandelt wie nicht reagierende Personen. Von einer Tuberkulinkonversion darf nur gesprochen werden, wenn eine Zunahme $\geq 10 \mathrm{~mm}$ in den letzten 2 Jahren erfolgte. Es ist empfohlen, Tuberkulinkonverter nach Ausschluss einer aktiven Erkrankung unabhängig von ihrem Alter präventiv zu behandeln; gut do- kumentiert ist die Wirksamkeit jedoch bei jüngeren Personen (ca. 60\% Risikoreduktion einer aktiven TB im 10-Jahres-Verlauf). Weniger Konsens besteht bei positiven Tuberkulinreaktionen von unklarer Dauer. Isoniazid über 9 Monate bleibt derzeit die Chemoprophylaxe der Wahl.

\section{(Din positiver Lymphozy- tenstimulationstest kann nicht zwischen aktiver und latenter TB unterscheiden; mit einem negativen Test kann eine aktive TB nicht ausgeschlossen werden}

Neu entwickelte Bluttests („in vitro T-cellbased interferon-g release assays", IGRAs) versprechen eine verbesserte Sensitivität und Spezifität zur Diagnose einer TB [6]. Vollblut (QuantiFERON-TB Gold ${ }^{\circ}$ ) oder aus Vollblut isolierte Lymphozyten (TSPOT.TB ${ }^{\circ}$ ) werden bei diesen Tests mit rekombinanten Peptiden („,early secretory antigen target-6“, ESAT-6; ,culture-filtrate-protein-10“, CFP-10) inkubiert. Diese Antigene kommen fast ausschließlich bei TB-Erregern vor. Antigenspezifische T-Zellen erkennen diese Peptide und produzieren Interferon-g, welches quantitativ gemessen wird. Vorteile dieser Tests sind ihre Unabhängigkeit von einer früheren BCG-Impfung, das Fehlen einer Kreuzreaktion mit Umweltmykobakterien, logistische Vorteile (nur ein Patientenbesuch nötig), Vermeidung subjektiver Ausmessungen und die Möglichkeit einer seriel- len Messung, ohne ein Boosterphänomen zu induzieren [17]. Ihre Rolle zur Evaluation eines individuellen Patienten mit möglicher aktiver TB-Erkrankung ist noch unklar. Die IGRAs basieren wie der Tuberkulintest auf einer zellulären Immunantwort, sodass die Unterscheidung zwischen einer latenten und aktiven Erkrankung nicht möglich sein wird. Dies ist problematisch, weil die meisten TB-Fälle in Populationen mit einer hohen TB-Prävalenz auftreten. Andererseits kann ein negativer IGRA eine TB-Infektion oder eine aktive Erkrankung nicht ausschließen. In einer kürzlich publizierten Studie hatten nur $64 \%$ der Patienten mit einer kulturell bestätigten TB einen positiven QuantiFERON-TB-Gold ${ }^{\circledR}$-Test [5]. In anderen Studien lag dieser Wert zwischen 55 und $88 \%$, gepoolt bei $75 \%$ [16], und damit ähnlich hoch wie die mittlere Sensitivität des Tuberkulintests. Es gibt zwar Hinweise, dass der T-SPOT.TB ${ }^{\circ}$ sensitiver ist als der QuantiFERON-TB Gold ${ }^{\otimes}[7,12]$, aber auch hier ist die Sensitivität $(80 \%)$ zu gering, um eine aktive Erkrankung bei einem negativen Test auszuschließen. Eine Erklärung für diese verminderte Sensitivität könnte die gut bekannte verminderte Immunantwort bei Patienten mit aktiver TB zum Zeitpunkt der Diagnosestellung sein, v. a. bei Patienten mit fortgeschrittenerer Erkrankung, Malnutrition oder höherem Alter.

Empfehlungen zum Einsatz von IGRAs zur Diagnose der latenten TB wurden von Expertengruppen des Royal College of Physicians [15] und der Lungenliga Schweiz [9] veröffentlicht. Beide Gremien empfehlen ein 2-Schritt-Verfahren:

1. Durchführung eines Tuberkulintests.

2. Bestätigung von positiven Tuberkulintests durch einen IGRA.

Bei immunsupprimierten Patienten sollte direkt ein IGRA durchgeführt werden.

\section{Diagnostik der pulmonalen Tuberkulose}

\section{Anamnese und Symptome}

Leitsymptom der Primärinfektion beim Erwachsenen ist das Fieber (70\%). Pulmonale Symptome wie Husten oder Auswurf sind nur bei einem Drittel vor- 
Hier steht eine Anzeige.

第 Springer 


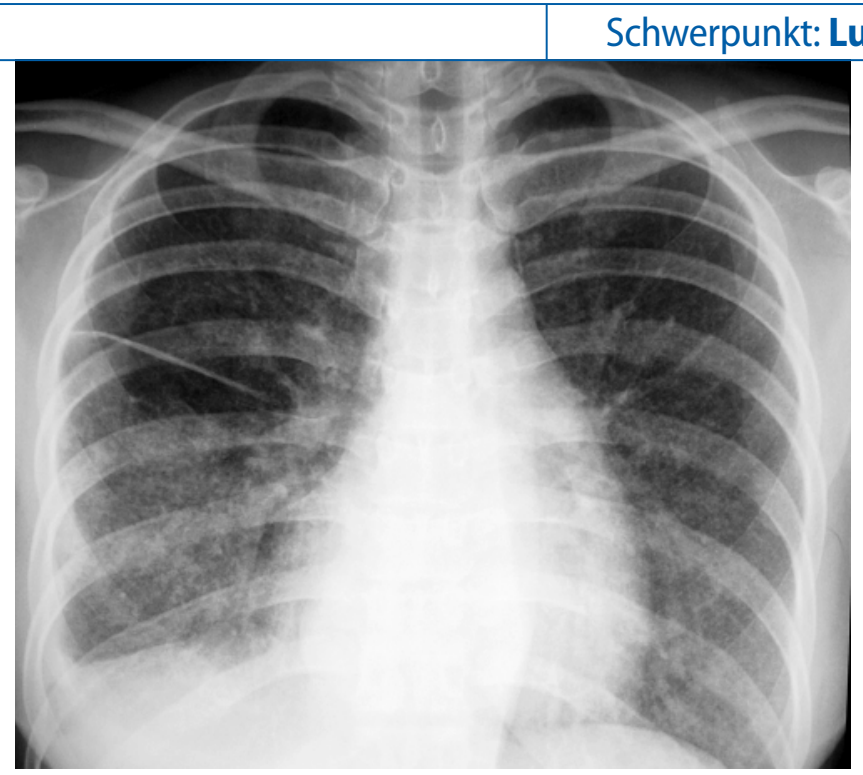

Abb. $2<$ Diffus feinnoduläres Infiltrat bei Miliartuberkulose

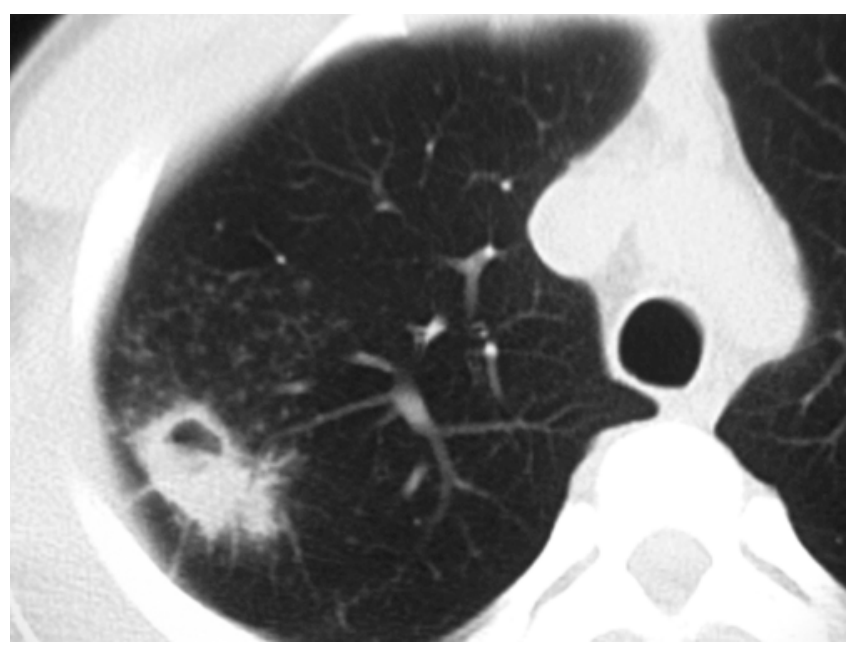

Abb. $3<$ Infiltrat mit Kaverne und bronchogener Streuung mit „Tree-in-bud"-Phänomen (luminale Impaktion kleiner, normalerweise nicht sichtbarer peripherer Bronchiolen)

handen; noch seltener sind pleuritische Schmerzen, Arthralgien und Müdigkeit. Die klinische Untersuchung ist meist unauffällig [18]. Die reaktivierten TB entwickeln sich meist langsam über Wochen bis Monate: Ein zu Beginn v. a. morgendlicher Husten (50-65\%) mit gelb-grünlichem Sputum nimmt im Verlauf an Intensität zu und wird oft erst bei bronchialer Mitbeteiligung als besorgniserregend empfunden. Eine meist milde Hämoptoe (25\%) entsteht durch endobronchiale Erosionen; eine massive Blutung durch Arrosion einer Pulmonalarterie ist heute sehr selten. In Niedrigprävalenzländern sind 5 bis $15 \%$ der Hämoptoen durch eine TB bedingt. Dyspnoe (33\%) entsteht durch eine zunehmende Beteiligung des Lungenparenchyms oder durch einen Pleuraerguss, selten durch einen komplizierenden Pneumothorax. Thoraxschmerzen (bis zu 33\%) sind Ausdruck einer pleuralen Beteiligung. Heiserkeit (laryngeale Beteiligung) und schmerzhafte pharyngeale oder gastrointestinale Ulcera sind heute selten. Unspezifische konstitutionelle Symptome treten mit fortschreitendendem Wachstum der Bakterienpopulation auf, sind langsam progredient und werden oft erstaunlich gut toleriert. Dazu gehören Müdigkeit (50-66\%), Gewichtsverlust (50$66 \%)$, Fieber (50\%) und Nachtschweiß (50\%).

\section{Klinische Befunde}

Die klinischen Befunde sind unspezifisch und abhängig vom Ausmaß der Progression der TB. Bei milder bis moderater Erkrankung wird oft ein Normalbefund erhoben. Im Allgemeinen wird das Ausmaß der Erkrankung durch die klinische Untersuchung unterschätzt. Immigranten beispielsweise, die bei Ankunft in ein Niedrigprävalenzland auf TB gescreent werden, sind mehrheitlich asymptomatisch mit einem normalen klinischen Befund und weisen trotz Vorliegen einer aktiven TB keine systemischen Entzündungszeichen auf [11]. Nichtkontinuierliche Rasselgeräusche können häufiger gehört werden, nachdem der Patient zuvor gehustet hat. Das Amphorenatmen als entfernt klingendes hohles Geräusch über Kavernen ist heute selten. Eine Klopfschalldämpfung mit abgeschwächtem Stimmfremitus weist auf eine pleurale Beteiligung hin.

\section{Radiologische Befunde}

Das Röntgenbild spielt eine zentrale Rolle in der TB-Diagnostik, sowohl zur Bestimmung des Ausmaßes der Erkrankung als auch zur Beurteilung des Therapieansprechens. Obwohl sie unspezifisch sind, müssen vor allem fleckige und noduläre Infiltrate im Oberlappen oder apikale Unterlappeninfiltrate mit assoziierter Lymphadenopathie und Kavernen an eine TB denken lassen. Bei Patienten mit gestörter zellvermittelter Immunität kann die Präsentation der TB atypisch sein und pulmonale Infiltrate können fehlen.

\section{( Bei Immunsupprimierten kann ohne radiologisch nachweisbares Infiltrat eine pulmonale TB vorliegen}

Bei der (selteneren) Primärinfektion ist eine hiläre Lymphadenopathie (65\%) am häufigsten. Sie ist nach 2 Monaten bei praktisch allen Personen vorhanden und heilt langsam (meist $>1$ Jahr) ab. Ein Drittel weist einen Pleuraerguss auf; pulmonale Infiltrate sind in $<30 \%$ nachzuweisen und nur selten progredient [18]. Die reaktivierte TB beginnt meist als asymmetrisch fleckige Pneumonie in den apikoposterioren Oberlappensegmenten (>80\%; Abb. 1). Kavernen sind in bis $\mathrm{zu} 40 \% \mathrm{zu}$ finden, Luftflüssigkeitsspiegel als Ausdruck einer schlechten Drainage oder einer intermittierenden Obstruktion in bis zu $20 \%$. Kleinere apikale Läsionen und Kavernen können mit Hilfe der Computertomographie zuverlässiger entdeckt werden. Bei der Miliartuberkulose liegt häufig ein diffuses feinnoduläres Infiltrat vor (• Abb. 2).

Exsudative (aktive) Infiltrate sind im Vergleich zu fibrotischen (inaktiven) nar- 
bigen Läsionen weicher, weniger scharf begrenzt und ändern sich im Verlauf. Ohne Verlauf ist es jedoch oft unmöglich, eine aktive TB von narbigen Veränderungen zu unterscheiden. Für eine bronchogene Streuung sprechen multiple, oft diskrete konfluierende Infiltrate, die selten progredient sind (• Abb. 3).

Obwohl von der WHO nicht empfohlen, ist eine radiologische Verlaufskontrolle (z. B. Röntgenthorax nach 2 Monaten und bei Abschluss der Therapie) nützlich zur Beurteilung des klinischen Ansprechens.

\section{Laborbefunde}

Auch hier gibt es keine spezifischen Befunde. Normalbefunde sind häufig bei einer pulmonalen TB. Mit zunehmender Erkrankung sind eine normochrome, normozytäre Anämie, eine Leukozytose (mit Monozytose in $<10 \%$ der Fälle) sowie eine Hypalbuminämie und Hypergammaglobulinämie zu erwarten. Eine Hyponatriämie kann Ausdruck eines SIADH bei tuberkulöser Meningitis oder einer Nebennierenrindeninsuffizienz sein, ist jedoch nicht selten bei einer isolierten pulmonalen TB zu finden. Bei der Miliartuberkulose sind die Transaminasen meist erhöht.

Der Pleuraerguss ist, falls vorhanden, typischerweise lymphozytär (meist 0,5$2,5 \times 10^{9}$ Leukozyten/l) mit erhöhtem Proteingehalt $(>25 \mathrm{~g} / \mathrm{l})$. Die Glukose ist oft erniedrigt, und der $\mathrm{pH}<7,3$. Direktpräparat und PCR aus der Pleuraflüssigkeit weisen eine enttäuschende Sensitivität auf; selbst die Kultur ist nur in 25 bis 30\% der Fälle positiv. Deutlich sensitiver ist die Pleurabiopsie, in der histologisch in $75 \%$ der Fälle Granulome nachweisbar sind. Obwohl bei etwa $30 \%$ kein pulmonales Infiltrat vorliegt, ist das Lungenparenchym fast immer mitbefallen.

\section{Mikrobiologische Diagnostik}

Obwohl in der Literatur umstritten, empfehlen wir eine sequenzielle Untersuchung dreier respiratorischer Materialien zur mikrobiologischen Diagnostik (Mikroskopie und Kultur; zusätzlich PCR v. a. bei oligosymptomatischen Patienten aus Hochendemieländern [10] und bei Immunsupprimierten). Die mikrobiologische Diagnos- tik muss in einer Sicherheitswerkbank der Klasse II durchgeführt werden, da die infektiöse Dosis (ca. 1o Mykobakterien) sehr tief ist und Mykobakterien lange in Aerosolen überleben können. Ist eine Sputumgewinnung nicht möglich, so sollte ein Reizsputum veranlasst werden. Dabei wird nach gründlichem Ausspülen des Mundes über 20 Minuten mit einer 3\%igen $\mathrm{NaCl}$ Lösung inhaliert und das gesamte in diesem Zeitraum gewonnene Sputum untersucht. Führt diese Methode nicht zum Ziel, so ist eine Probengewinnung mittels einer bronchoalveolären Lavage indiziert.

Die Mikroskopie ist ein schnelles und billiges Verfahren, um eine TB zu diagnostizieren und hoch kontagiöse Patienten zu erkennen. Heutzutage wird nach einer Vorbehandlung meist ein Screening mit einer Fluoreszenzfärbung ( $\mathrm{Au}$ ramin-Rhodamin) durchgeführt, da die grün fluoreszierenden Mykobakterien auf schwarzem Grund besser sichtbar sind als die säurefesten Stäbchen in der anschließend als Bestätigungstest durchgeführten Ziehl-Neelsen-Färbung. Materialien mit $10^{6}$ säurefesten Stäbchen (SFS) sind zuverlässig mikroskopisch positiv, bei $10^{4} \mathrm{SFS}$ beträgt die Sensitivität nur noch 60\%. Die Spezifität der Mikroskopie ist hoch; ihre Aussagekraft unter Therapie ist jedoch limitiert, da Patienten über längere Zeit tote Bakterien auswerfen, obwohl die Kulturen längst negativ geworden sind [22].

Mit Hilfe molekularer Methoden kann die Diagnose einer TB schneller gestellt werden. Notwendig für ein positives PCRResultat sind etwa $10^{2}\left(-10^{3}\right)$ SFS. Für mikroskopisch positive Materialien weist die PCR eine praktisch $100 \%$ ige Sensitivität und Spezifität auf, für mikroskopisch negative Materialien ist die Sensitivität vermindert (40-80\%). Somit ist auch diese Untersuchung nicht zum sicheren Ausschluss einer TB geeignet.

Zur Verlaufskontrolle unter Therapie eignet sich diese Technik nicht. Aufgrund des hohen positiv prädiktiven Werts einer positiven Mikroskopie ist die PCR-Untersuchung bei typischer Klinik eigentlich nicht nötig. Im klinischen Alltag ist sie jedoch hilfreich als schneller Bestätigungstest und bei Immunsupprimierten, bei denen differenzialdiagnostisch an nichttuberkulöse Mykobakterien gedacht werden muss.
Internist 2007 · 48:489-496

DOI 10.1007/s00108-007-1814-0

๑) Springer Medizin Verlag 2007

\section{G. Laifer · S. Bassetti \\ Diagnostik der pulmonalen Tuberkulose beim Erwachsenen}

\section{Zusammenfassung}

Die Inzidenz der pulmonalen Tuberkulose (TB) in Westeuropa nimmt ab. Dennoch ist sie vor allem bei Migranten und Immunsupprimierten (z. B. HIV) keine seltene Erkrankung. Bei Immunsupprimierten ist die Präsentation oft atypisch; generell unterschätzt die Klinik das Ausmaß der Erkrankung. Eine radiologische Abklärung und eine Untersuchung von 3 sequenziell gewonnenen Sputa inklusive Mikroskopie, Kultur und Resistenztestung auf die Erstlinienmedikamente sind nötig zur Diagnosestellung. Der Tuberkulintest kommt vor allem bei der Diagnose der latenten TB und bei Umgebungsabklärungen zum Einsatz. Neuere Bluttests, basierend auf einem Nachweis von Interferon-g, welches durch antigenspezifische T-Zellen gebildet wird, haben gewisse Vorteile gegenüber dem Tuberkulintest.

\section{Schlüsselwörter}

Diagnostik - QuantiFERON-TB · T-SPOT.TB . Tuberkulintest $\cdot$ Tuberkulose

\section{Diagnostics for pulmonary tuberculosis in adults}

\section{Abstract}

The incidence of pulmonary tuberculosis (TB) is decreasing in Western Europe. However, TB should not be considered to be a rare disease, particularly in immigrants and in immunocompromised persons (i.e. HIV infection). The clinical presentation is often atypical in immunocompromised persons. In general, the extent of the disease is underestimated by the clinical presentation. X-ray and a sequential investigation of three samples of sputum including microscopy, culture and susceptibility testing for the first-line drugs should be obtained. Tuberculin testing is useful in the diagnosis of latent TB and in screening persons with close contact to patients with active disease. New blood tests based on the detection of interferon-g released by antigen specific T-cells have some advantages compared to tuberculin testing.

\section{Keywords}

Diagnosis · QuantiFERON-TB · T-SPOT.TB . Tuberculin testing $\cdot$ Tuberculosis 


\begin{tabular}{|c|c|c|}
\hline Methode & $\begin{array}{l}\text { Anzahl SFS* für } \\
\text { positives Resultat }\end{array}$ & Kommentar \\
\hline Mikroskopie & $\begin{array}{l}\geq 10^{6}(100 \% \text { positiv }) \\
10^{4} \text { (60\% positiv) }\end{array}$ & $\begin{array}{l}\text { Schnelltest ( } \leq 24 \mathrm{~h} \text { ) } \\
\text { Kostengünstig } \\
\text { Identifiziert hoch kontagiöse Patienten } \\
\text { Geringe Sensitivität } \\
\text { Hohe Spezifität } \\
\text { Fluoreszenztest als Screeningtest sensitiver als Ziehl- } \\
\text { Neelsen-Färbung (positiver Fluoreszenztest sollte mit } \\
\text { Ziehl-Neelsen-Färbung verifiziert werden) }\end{array}$ \\
\hline PCR & $10^{2}\left(-10^{3}\right)$ & $\begin{array}{l}\text { Schnelltest (6-48 h, je nach Infrastruktur) } \\
\text { Sensitivität } 95-100 \% \text { falls mikroskopisch positiv, } \\
40-80 \% \text { falls mikroskopisch negativ } \\
\text { Hohe Spezifität ( } 95-100 \%) \\
\text { Nicht geeignet für Verlaufskontrollen }\end{array}$ \\
\hline $\begin{array}{l}\text { Kultur (bleibt } \\
\text { Goldstandard } \\
\text { der mikro- } \\
\text { biologischen } \\
\text { Diagnostik) }\end{array}$ & $10^{1}-10^{2} / \mathrm{ml}$ & $\begin{array}{l}\text { Langsamer Test ( } 8-24 \text { Tage je nach Test und Erregerzahl) } \\
\text { Sensitivität }>90 \% \text { bei Kombination von festen und flüs- } \\
\text { sigen Medien } \\
\text { Nötig zur Speziesidentifikation und Resistenztestung } \\
\text { Kontrolle des Therapieerfolgs möglich }\end{array}$ \\
\hline
\end{tabular}

Goldstandard der mikrobiologischen Diagnostik bleibt die Kultur, die die Resultate des Direktnachweises (Mikroskopie, PCR) bestätigt und die Biomasse für die Identifizierung und Resistenzprüfung liefert sowie den Therapieerfolg dokumentiert. In $10(-20) \%$ aller Fälle wird jedoch eine kulturnegative TB vorliegen. Diese besteht, wenn ohne dokumentiertes Wachstum aufgrund der klinischen, radiologischen und epidemiologischen $\mathrm{Be}$ funde eine TB hoch wahrscheinlich ist, keine Alternativdiagnose gefunden wird und ein klinisches und radiologisches Ansprechen auf eine 6- bis 8-wöchige empirische Therapie vorliegt. Hauptgründe für eine kulturnegative TB sind eine nicht adäquate Sputumgewinnung oder eine tiefe Konzentration an Mykobakterien. Für eine positive Kultur sind $10^{1}$ bis $10^{2} \mathrm{SFS} / \mathrm{ml}$ nötig. Die Kombination von festen und flüssigen Medien erhöht die Sensitivität der Kultur auf etwa 90\%. Damit ist die Kultur der Mikroskopie und der PCR überlegen. Feste Kulturmedien auf Eibasis (z. B. Löwenstein-Jensen) zeigen ein sichtbares Wachstum von $M$. tuberculosis nach 18 bis 24 Tagen. Agarmedien (z. B. Middlebrook $7 \mathrm{H} 10$ oder $7 \mathrm{H} 11$ ) sind besser standardisiert und zeigen ein schnelleres Wachstum (1014 Tage). Flüssigmedien (z. B. Septi-Chek System; radiometrische BACTEC-46oTBMethode) sind die schnellsten Methoden (Wachstum nach 8-14 Tagen). Derzeit sind verbesserte nichtradiometrische Systeme erhältlich, welche ein kontinuierliches Monitoring von Mykobakterienwachstum erlauben. Vor dem Beimpfen von Nährmedien müssen respiratorische Materialien homogenisiert, dekontaminiert und aufkonzentriert werden. Dies erklärt den erhöhten Zeitbedarf für die mykobakterielle Diagnostik.

Eine Zusammenfassung der wichtigsten Charakteristika der einzelnen Tests zeigt • Tab. 2.

Mit Hilfe von Gensonden ab Kultur kann M. tuberculosis-Komplex von nichttuberkulösen Mykobakterien abgegrenzt werden. Diese Technik weist ab Kultur eine sehr hohe Spezifität und nahezu 100\%ige Sensitivität auf; die Resultate liegen innerhalb von $2 \mathrm{~h}$ vor.

Moderne molekulare Typisierungsverfahren werden vor allem bei epidemiologischen Fragestellungen eingesetzt wie, z. B. zur Aufklärung von Übertragungsketten. Außerdem kann mit ihnen eine genaue Identifizierung innerhalb des $M$. tuberculosis-Komplexes erfolgen, und Subtypen von M.tuberculosis (z. B. PekingKlon oder Harlem-Typ) können identifiziert werden.

\section{Resistenzprüfung}

Besorgniserregend ist das Auftreten multiresistenter Stämme (definiert als Resis- tenz auf Isoniazid und Rifampicin) mit einer Prävalenz von etwa 3\% (WHO-Schätzung) und hohen Raten vor allem in Osteuropa, Israel und China [2]. Daneben ist eine zunehmende Isoniazidresistenz (ca. $10 \%) \mathrm{zu}$ verzeichnen, sowie eine weltweite Ausbreitung von multiresistenten Stämmen, welche zusätzlich zur Resistenz auf Isoniazid und Rifampicin eine Resistenz auf Fluorochinolone und auf Reserve-TBMedikamente (wie Capreomycin, Kanamycin, Amikacin) aufweisen („extensive resistant TB“; [13]). Deshalb ist heute eine Resistenzprüfung auf die Erstlinienmedikamente für jedes Erstisolat nötig.

\section{(2) Eine Resistenztestung auf alle Erstlinienmedikamente ist heute aufgrund der zunehmenden Resistenz- entwicklung obligat}

Bleibt die Kultur im Verlauf über längere Zeit positiv, so ist eine Wiederholung der Resistenzprüfung nach spätestens 3 Monaten indiziert, um das Auftreten möglicher Resistenzen zu überwachen. Weit verbreitet sind die radiometrische BACTEC460TB-Methode und die Proportionsmethode. Sie wird auf Middlebrook-Agar durchgeführt und gilt noch als Goldstandard. Ergebnisse sind in etwa 3 Wochen verfügbar. Eine neue, preiswerte kulturbasierte Methode zum schnelleren Nachweis (inklusive der multiresistenten TB) ist der MODS-Assay („microscopic observation drug susceptibility assay“), welcher auf einer frühen mikroskopischen Erkennung der typischen Zopfformationen von M. tuberculosis beruht. In einer kürzlich publizierten Studie war dieser Test sensitiver als die Vergleichsmethoden mit einer kürzeren Zeit bis zur positiven Kultur (Median 7 Tage; [14]).

Ein Einsatz molekularer Technologien zur Resistenztestung ist noch nicht Routine. Bei dringendem Verdacht auf eine Rifampicinresistenz kann der Einsatz molekularer Techniken erwogen werden, da $>95 \%$ dieser Stämme eine Mutation in einem sehr kurzen Genabschnitt des rpoB-Gens aufweisen [20]. Für andere Medikamente ist die Situation komplizierter, da Mutationen häufig in verschiedenen Genen lokalisiert sind [23]. 
Hier steht eine Anzeige.

第 Springer 


\section{Verlaufskontrolle}

Zur Verlaufskontrolle ist eine monatliche Sputumuntersuchung (Direktpräparat und Kultur) empfohlen, bis 2 konsekutive Sputa kulturell negativ sind [3].

\section{- Die PCR-Diagnostik auf $M$. tuberculosis-Komplex eignet sich nicht für Verlaufsuntersuchungen.}

Sind Direktpräparat und Kultur nach 2 Monaten noch positiv, so hat dies die Konsequenz einer Verlängerung der Therapiedauer von 6 auf 9 Monate. Ist das Direktpräparat nach dem 5 . Monat noch positiv, wird gemäß WHO von einem Therapieversagen ausgegangen und ein Wechsel der Behandlungsstrategie nötig.

\section{Fazit für die Praxis}

Die TB ist weltweit sehr häufig, zunehmend mit resistenten Keimen. Migranten aus Hochendemiegebieten haben das Risiko ihres Herkunftslandes. Diagnostisch wegweisend ist das Röntgenbild, jedoch muss bei Immunsupprimierten bei atypischer Präsentation oder fehlendem Infiltrat an eine TB gedacht werden. 3 sequenziell abgenommene Sputa sind empfohlen (Mikroskopie und Kultur, evtl. PCR auf M. tuberculosis-Komplex). Goldstandard bleibt die Kultur, die für die Sensibilitätstestung auf die Erstlinienmedikamente nötig ist. Eine Diagnostik für eine latente TB soll nur bei Personen mit erhöhtem TB-Risiko, erhöhtem beruflichen Risiko und Kontaktpersonen mit offener TB vorgenommen werden und sollte im Falle eines positiven Befundes nach Ausschluss einer aktiven Erkrankung eine präventive Therapie zur Folge haben. Tuberkulintest und neu entwickelte Bluttests basierend auf einem Nachweis von Interferon-g nach Ex-vivo-Exposition mit mykobakteriellen Antigenen sind nach heutigem Erkenntnisstand im wesentlichen äquivalent mit gewissen Vorteilen für die neueren Bluttests.

\section{Korrespondierender Autor \\ Dr. G. Laifer}

Klinik für Infektiologie, Universitätsspital Basel Petersgraben 4, CH-4031 Basel

Laiferg@uhbs.ch

Interessenkonflikt. Es besteht kein Interessenkonflikt. Der korrespondierende Autor versichert, dass keine Verbindungen mit einer Firma, deren Produkt in dem Artikel genannt ist, oder einer Firma, die ein Konkurrenzprodukt vertreibt, bestehen. Die Präsentation des Themas ist unabhängig und die Darstellung der Inhalte produktneutral.

\section{Literatur}

1. American Thoracic Society (2000) Targeted tuberculin testing and treatment of latent tuberculosis infection. MMWR 49: 1-51

2. Aziz MA, Wright A, Laszlo A et al. (2006) Epidemiology of antituberculosis drug resistance (the Global Project on Anti-tuberculosis Drug Resistance Surveillance): an updated analysis. Lancet 368 : 2142-2154

3. Centers for Disease Control and Prevention (2003) Treatment of tuberculosis. MMWR Recomm Rep 52:1-77

4. Corbett EL, Watt CJ, Walker N et al. (2003) The growing burden of tuberculosis: global trends and interactions with the HIV epidemic. Arch Intern Med 163: 1009-1021

5. Dewan PK, Grinsdale K, Kawamura LM (2007) Low sensitivity of a whole-blood interferon-g release assay for detection of active tuberculosis. Clin Infect Dis 44: 69-73

6. Ewer K, Deeks J, Alvarez L et al. (2003) Comparison of T-cell-based assay with tuberculin skin test for diagnosis of Mycobacterium tuberculosis infection in a school tuberculosis outbreak. Lancet 361 : 1168-1173

7. Ferrara G, Losi M, D'Amico R et al. (2006) Use in routine clinical practice of two commercial blood tests for diagnosis of infection with Mycobacterium tuberculosis: a prospective study. Lancet 367: 1328-1334

8. Frieden TR, Sterling TR, Munsiff SS et al. (2003) Tuberculosis. Lancet 362: 887-899

9. Kommission „Interferon-Gamma für die Diagnostik der Tuberkulose-Infektion“ der Lungenliga Schweiz (2005) Erkennung der Tuberkuloseinfektion mittels Bluttest (Interferon-gamma). Bundesamt für Gesundheit Bulletin 45: 822-823

10. Laifer G, Widmer AF, Frei R et al. (2004) Polymerase chain reaction for Mycobacterium tuberculosis: impact on clinical management of refugees with pulmonary infiltrates. Chest 125: 981-986

11. Laifer G, Widmer AF, Simcock M et al. (2007) Pulmonary tuberculosis in a low incidence country: new immigrants, foreign-born residents and native residents. Am J Med (in press)

12. Lee JY, Choi HJ, Park IN, et al. (2006) Comparison of two commercial interferon-gamma assays for diagnosing Mycobacterium tuberculosis infection. Eur Resp J 28: 24-30

13. Morbidity and Mortality Weekly Report (2006). Emergence of Mycobacterium tuberculosis with extensive resistance to second-line drugs worldwide 2000-2004. 55: 301-305

14. Moore DA, Evans CA, Gilman RH et al. (2006) Microscopic-observation drug-susceptibility assay for the diagnosis of tuberculosis. N Engl J Med 355 : $1539-1550$
15. National Collaborating Centre for Chronic Conditions (2006) Tuberculosis: clinical diagnosis and management of tuberculosis, and measures for its prevention and control. Royal College of Physicians, London

16. Pai M, Menzies D (2007) Interferon-g release assays: what is their role in the diagnosis of active tuberculosis? Clin Infect Dis 44: 74-77

17. Pai M, Riley LW, Colford JM Jr (2004) Interferongamma assays in the immunodiagnosis of tuberculosis: a systematic review. Lancet Infect Dis 4: 761-776

18. Poulsen A (1957) Some clinical features of tuberculosis. Acta Tuberc Scand 33: 37-92

19. Small PM, Fujiwara PI (2001) Management of tuberculosis in the United States. N Engl J Med 345: 189-200

20. Telenti A, Imboden P, Marchesi F et al. (1993) Detection of rifampicin-resistance mutations in Mycobacterium tuberculosis. Lancet 341: 647-650

21. Tissot F, Zanetti G, Francioli P et al. (2005) Influence of bacille Calmette-Guerin vaccination on size of tuberculin skin test reaction: to what size? Clin Infect Dis 40: 211-217

22. Vidal R, Casabona N, Juan A et al. (1996) Incidence and significance of acid-fast bacilli in sputum smears at the end of antituberculous treatment. Chest 109: 1562-1565

23. Zhang $Y$, Vilchèze $C$, Jacobs WR et al. (2005) Mechanisms of drug resistance in Mycobacterium tuberculosis. In: Cole ST, Eisenach KD, McMurray DN et al. (Eds) Tuberculosis and the tubercle bacillus. ASM Press, Washington DC, p 115-140 